\title{
Temperature Measurement Method by Infrared Radiation Pyrometer Using Chopped Broadband Radiation - Signal Conditioning and Acquisition Elements Design
}

\author{
K. T. Aminu, D. D. Yakubu, S.A. Baraza, B. D. Halilu \\ Department of Electrical Engineering \\ Abubakar Tatari Ali Polytechnic Bauchi \\ Nigeria
}

\begin{abstract}
In this paper, we report the design of a signal conditioning and acquisition elements of a chopped broadband radiation pyrometer for measuring temperature in the range of $900{ }^{\circ} \mathrm{C}$ to $1200^{\circ} \mathrm{C}$. We also report the selection of a suitable chopping frequency within the physical properties of the system and provided the calibration characteristics for the pyrometer. Based on the results, it was found that the designed signal conditioning circuit was able to produce an output voltage of $0.24-0.60 \mathrm{~V}$ corresponding to the temperature measuring range of the pyrometer. This was achieved through the use of a phase-sensitive detector (PSD) in the form of a lock-in amplifier in combination with a low pass filter. A suitable chopping frequency of $30 \mathrm{~Hz}$ was chosen for the shutter pulse so that the detector can have sufficient time to settle before the next shutter pulse transition. Finally, the result also shows that the calibration characteristic of the pyrometer presumes an approximately linear relationship.
\end{abstract}

Keywords: Contactless temperature sensor, Pyrometer, Infrared, Chopped Broadband, Signal Conditioning, Detector, Lock-In Amplifier.

\section{INTRODUCTION}

High temperature measurements of objects are needed in many technical and research applications. For technical applications, however, the pyrometer should be simple, low cost, easy to handle and suitable for temperature measurements with reasonable accuracy. The primary focus of the present study is the design of signal conditioning circuit and acquisition elements for a noncontact temperature measurement of objects using chopped broadband radiation pyrometer. In recent years, the chopped broadband radiation pyrometer has been used for high temperature measurements because of its unique feature. This feature is a mechanical shutter that closes and opens the gate to the detector intermittently to prevent it from being burnt due to high temperature [1-2]. However, there are other techniques of high temperature measurement currently in use: narrow band radiation pyrometer and the broadband (unchopped) radiation pyrometer [3]. All these systems of temperature measurement operate on the same principle. However, in contrast, the chopped broadband radiation pyrometer differs from the traditional systems only because of the peculiar feature [1]. The errors of temperature measurements using classic approaches are often relatively high and it is very difficult to estimate such errors. These errors are due to the actual surface condition of the object, the effect of oxidation and the coating of the black body [2] [4]. The main purpose of the broadband radiation pyrometers is that they can potentially bring significant improvement in accuracy in non-contact temperature measurements, particularly in the case of so called difficult objects. These objects are considered to be the ones whose emissivity depends on the wavelength and time. Such cases can be found in many technical applications, and particularly often in the steel and semiconductor industry [4]. Additionally, the measurement accuracy of the system can be increased by reducing the influence of background temperature of the optical system. Hence, the need for conditioning the information acquired [2]. In recent years, there has been an increasing amount of literature on infrared pyrometer temperature measurement system [2] [4-8].

A brief background of infrared systems for non-contact temperature measurement, using the radiation emitted by the object under test, is presented in Section 1. The basic theory of the radiation pyrometer and the detector is presented in Section 2. The design details are presented in Section 3. Results obtained using the design parameters are given in section 4, and conclusions are drawn in Section 5. 
International Journal of Advances in Scientific Research and Engineering (ijasre), Vol 6 (11), November -2020

\section{THEORY}

\section{A. Basic Theory}

All bodies emit electromagnetic radiation as a function of their temperature above absolute zero. The ideal emitter is termed a black body: from Planck's law, the spectral existence of radiation emitted by a black at a temperature $T \mathrm{~K}$ is [5]:

$$
W^{B B}(\lambda, T)=\frac{C_{1}}{\lambda^{5}\left[\exp \left(\frac{C_{2}}{\lambda T}\right)-1\right]}
$$

where $W^{B B}(T, \lambda)$ is the hemispherical spectral radiant intensity $C_{I}$ and $C_{2}$ are Plank's constants with values of $37,413 \mathrm{~W} \mu \mathrm{m}^{4} / \mathrm{cm}^{2}$ and $14,388 \mu \mathrm{m} \mathrm{K}$ respectively, $\lambda$ is the wavelength of radiation in $\mu \mathrm{m}$, and $T$ the absolute temperature of the blackbody in degree Kelvin. However, the Wien's approximation is given by [5]:

$$
W^{B B}(\lambda, T)=\frac{C_{1}}{\lambda^{5} \exp \left(C_{2} / \lambda T\right)}
$$

can be used with an error of $10 \%$ for values of $\lambda T<3125 \mu \mathrm{m} \mathrm{K}$. A correlation for the maximum of the spectral radiation energy can be derived from Equation (1), which is called the Wien's displacement law:

$$
\lambda_{\max } T=2897.8 \mu m K
$$

However, in some cases, the influence of emissivity variations can be reduced tremendously by the use of two-color fiber-optic pyrometers measuring the emitted radiation at two specific wavelength bands [5].

\section{i. Spectral radiation pyrometer}

An equation for the total rate of radiation emitted per second is given by [4] [9]:

$$
P_{D}=K T^{4}\left(W m^{-2} K^{4}\right)
$$

where $T$ is the absolute temperature of the body and $K$ is a constant of radiation that depends on the heat intensity, the nature of the heat spectrum and the kind of material of the body. For this application $K$ is taken as 50 for the detector material. The power spectral density of this emission varies with temperature in the manner shown below. The major part of the frequency spectrum lies within the band of wavelengths between $0.3 \mu \mathrm{m}$ and $40 \mu \mathrm{m}$, which corresponds to the visible and infrared ranges. As the magnitude of the variation varies with the temperature, measurement of the emission from a body allows the temperature to be calculated. Choice of the best method of measuring the emitted radiation depends on the temperature of the body. At low temperatures, the peak of the power spectral density function lies in the infrared region, whereas at higher temperatures it moves towards the visible part of the spectrum. This phenomenon is observed as the red glow which a body begins to emit as its temperature is increased beyond $600{ }^{\circ} \mathrm{C}[9]$.

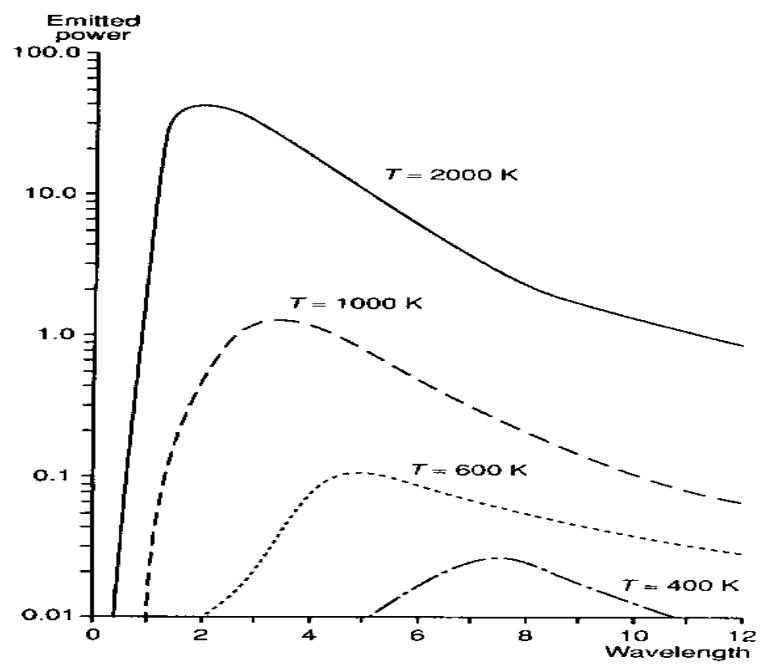

Figure 1: Radiation spectrum of an object heated at different temperature [9] 


\section{ii. Pyrometer}

All the alternative forms of radiation pyrometer have an optical system which is similar to that in the optical pyrometer and focuses the energy emitted from the measured body. They differ, however, by omitting the filament and eyepiece and using instead an energy detector in the same focal plane as the eyepiece was. The radiation detector is either a thermal detector, which measures the temperature rise in a black body at the focal point of the optical system or a photon detector. Thermal detectors respond equally to all wavelengths in the frequency spectrum whereas photon detectors respond selectively to particular band within the full spectrum [9].

Radiation thermometers have one advantage in that they do not require being in contact with the hot body in order to measure its temperature. Thus, there is no disturbance at all in the measured system. Furthermore, there is no possibility of contamination, which is particularly important in the food industries and other process industries. They are also capable of measuring the temperature of moving bodies, for instance the temperature of steel bars in rolling mill [7] [9].

\section{iii. The chopped broad- band radiation pyrometer}

A number advantages accrue when the radiation coming from the target to the detector is interrupted periodically (chopped) at a fixed frequency; therefore many infrared systems employ this technique. When high sensitivity is needed, amplification is required, and high gain ac amplifiers are easier to construct than their dc counterparts. This is usually the main reason for using choppers. Additional benefits related to ambient- temperature compensation and reference-source comparison also may be obtained. Systems employing thermal (broadband) and photon (restricted-band) detectors and choppers are in common use. Here in this application special consideration is given to thermal detectors [9].

\section{iv. Thermal detectors}

Thermal detectors are blackened elements designed to absorb a maximum of the incoming radiation at all wavelengths. The absorbed radiation causes the temperature of the detector to rise until equilibrium is reached with heat losses to the surroundings. In order to examine the factors affecting both the sensitivity and time constant of a thermal detector, we now calculate the transfer function relating detector temperature $\mathrm{T}_{\mathrm{D}}$ to power $\mathrm{P}_{\mathrm{D}}[4][9]$. The heat balance equation for the detector is:

$$
P_{D}-U A\left(T_{D}-T_{S}\right)=M C \frac{d T_{D}}{d t}
$$

where $T_{D}$ is the total incident power on the detector, $T_{S}$ is the surrounding temperature, $M$ detector mass in $\mathrm{kg}, C$ detector specific heat in $\mathrm{J} \mathrm{kg}^{-1}{ }^{\circ} \mathrm{C}^{-1}, A$ detector surface area in $\mathrm{m}^{2}$, and $U$ represents the heat transfer coefficient in $\mathrm{W} \mathrm{m}{ }^{-2} \mathrm{C}^{-1}$.

Rearranging Equation (5), we have:

$$
T_{D}+\tau \frac{d T_{D}}{d t}=P_{D} / U A+T_{S}
$$

where time constant $\tau=\frac{M C}{U A}$. To obtain steady state solution, we set $\frac{d T_{D}}{d t}=0$,

$$
T_{D}=P_{D} /_{U A}+T_{S}
$$

This result is intuitively reasonable since it says that $T_{D}$ is proportional to $P_{D}$, the rate of heat influx and inversely proportional to $U A$, which governs the rate of heat loss [9].

\section{PYROMETER SYSTEM DESIGN}

A block diagram of the pyrometer system design is illustrated in Figure 2. It includes the radiation source (hot object whose temperature is to be measured), the optical filter, and the radiation/photodetector with corresponding lock-in amplifier and low pass filter, thus performing the signal conditioning. The active element close to the measuring unit corresponds to the optic filter. It is mechanically powered by the rotating shutter and driven by an electrical square signal to perform an on/off control. The mechanical rotating shutter is for switching of the chopped spectral bands simultaneously. 


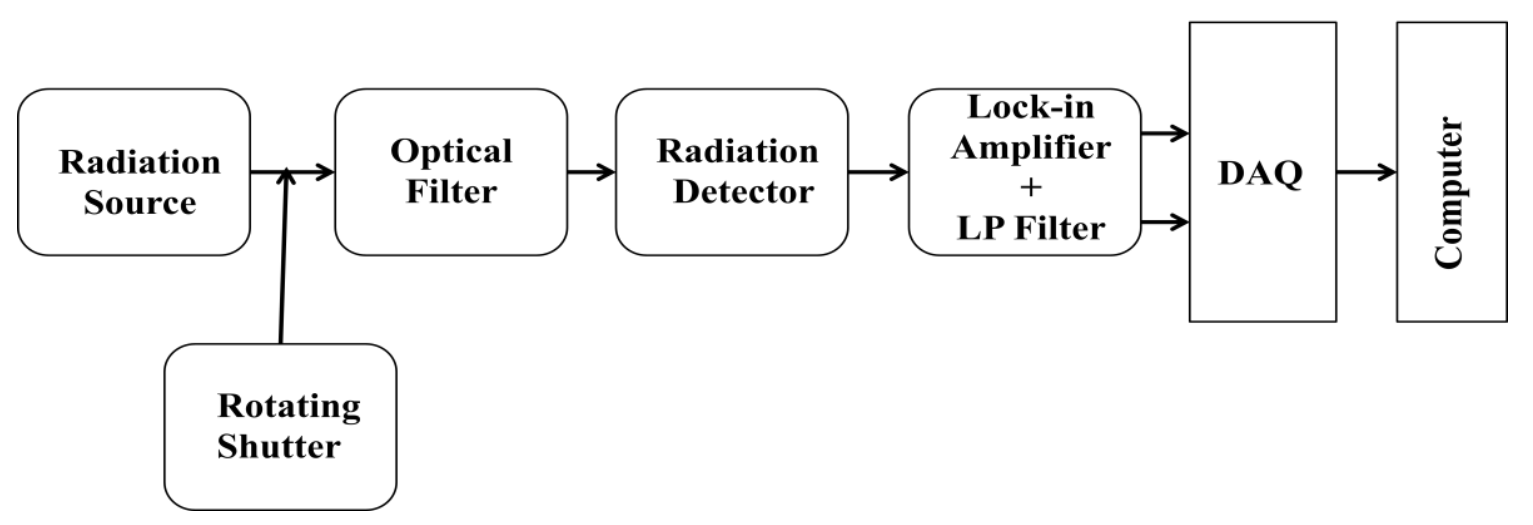

Figure 2: Schematic of the pyrometer system design with mechanically powered rotating shutter

The signal processing system (Figure 2) is designed to be able to modulate the optical signal (on/off control) and to demodulate the radiation emitted by the hot object so as to significantly reduce the effect of environmental noise. The photodetector with highgain transimpedance amplifier is used to convert the receiving light from radiation to an output voltage. The optical filter is placed close to the hot object and before the photodetector to minimise the influence of atmospheric absorption on a signal from the object to the detector. The detector absorbs radiation whose wavelength is shorter than the cut-off wavelength. Absorption of such radiation causes an increase in electrical conductivity of the material and corresponding decrease in the detector resistance. This effect enables the system to measure radiation reaching the detector [8].

Information about the radiation signals measured in the system from the PSD (lock-in amplifier + low pass filter) through the data acquisition (DAQ) system to personal computer (PC). A software application is usually developed to control the communication process between the DAQ and the PC, to carry out calculations, calibration, and visualisation of the measurement results.

\section{i. Mechanical Rotating Shutter Design}

The total radiation released per unit surface area of a black body is given according to the Stefan-Boltzman law as given in Equation (4). The time constant and chopping frequency of the thermal detector governs the speed of the mechanical rotating shutter. The time constant is given by:

$$
\tau=\frac{M C}{U A}
$$

The chopping frequency of the rotating shutter is also given by:

$$
f_{c}=\frac{1}{2 \pi \tau}
$$

\section{ii. The Thermal Detector Design}

The bolometer is commonest form of thermal detectors and it is the kind used in this application. It is a metal or semiconductor (thermistor) resistance material in the form of films or flakes which are blackened to maximize the absorption of the incoming radiation. The incident power $P_{D}(W)$ would heat the detector to a temperature $T_{D}\left({ }^{\circ} \mathrm{C}\right)$ which is usually above the surrounding temperature $T_{S}\left({ }^{\circ} \mathrm{C}\right)$ [1]. The heat balance equation for the detector is given by Equation (5). By equation (7), a relationship has been established that gives the intercept as $T_{S}$ and the gradient as the inverse of the product $U A$ when presented graphically.

Since the bolometer is a passive resistive device, a bridge circuit can be used to produce an output voltage proportional to temperature. Two sensors, labeled $R_{1}$ and $R_{2}$ in Figure 3 are the measuring and reference elements (in this application $T_{D}$ and $T_{S}$ respectively) [9]. 


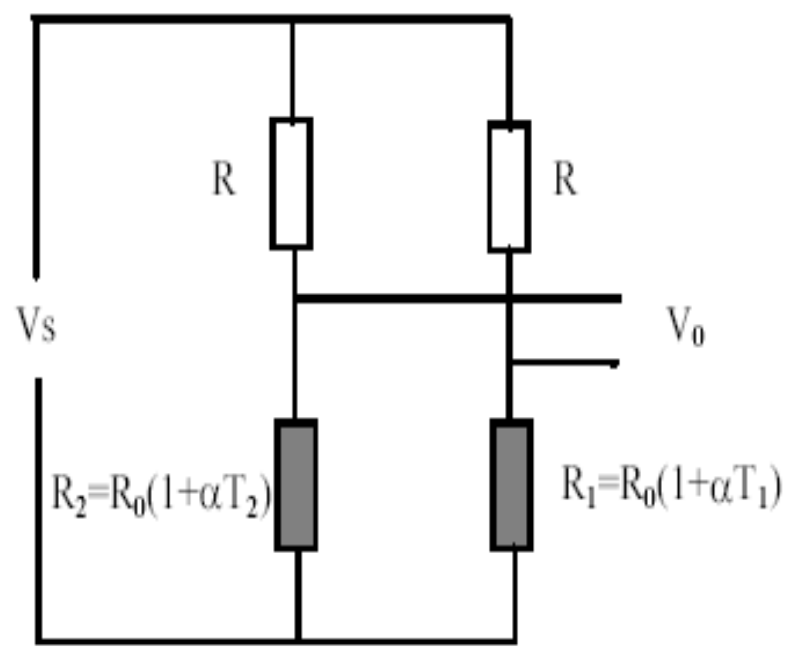

Figure 3: Bridge circuit of a bolometer sensor [9]

From the circuit in Figure 3:

$R_{1}$ is the measuring element at the temperature $T_{D}\left({ }^{\circ} \mathrm{C}\right)$ and is given by:

$$
R_{1}=R_{0}\left(1+\alpha T_{D}\right)
$$

$R_{2}$ is the reference element at the surrounding temperature $T_{S}\left({ }^{\circ} \mathrm{C}\right)$ and is equally given by:

$$
R_{2}=R_{0}\left(1+\alpha T_{s}\right)
$$

$R_{0}$ in Equation (10) and Equation (11) is the internal resistance of the bolometer (sensor) at temperature $0{ }^{\circ} \mathrm{C}$ and $\alpha$ is the temperature coefficient of resistance. The two $R$ 's in the circuit are fixed value resistors. The output voltage of the bridge circuit can be approximated by:

$$
V_{o}=V_{s}\left(\frac{R_{o}\left(1+\alpha T_{D}\right)}{R+R_{o}\left(1+\alpha T_{D}\right)}-\frac{R_{o}\left(1+\alpha T_{S}\right)}{R+R_{o}\left(1+\alpha T_{S}\right)}\right)
$$

Rearranging Equation (12) yields:

$$
V_{o}=V_{S}\left(\frac{1}{1+\frac{R}{R_{o}\left(1+\alpha T_{D}\right)}}-\frac{1}{1+\frac{R}{R_{o}\left(1+\alpha T_{S}\right)}}\right)
$$

If $R$ is chosen to be much greater than $R_{o}$ (i.e $R>>R_{o}$ ) then:

$$
V_{o} \approx V_{S}\left(\frac{1}{\frac{R}{R_{o}\left(1+\alpha T_{D}\right)}}-\frac{1}{\frac{R}{R_{o}\left(1+\alpha T_{S}\right)}}\right)
$$

Following some simplifications, the output of the bolometer can be obtained from Equation (15).

$$
V_{o} \approx V_{S} \alpha \frac{R_{o}}{R}\left(T_{D}-T_{S}\right)
$$


Substituting Equation (7) in Equation (15) yields:

$$
V_{o} \approx \frac{V_{s} \alpha R_{o}}{U A R} P_{D}
$$

In Equation (16), $V_{o}$ is the output voltage (v), $V_{S}$ represents the supply voltage (v), $\alpha$ denotes the temperature coefficient of resistance $\left(\mathrm{C}^{-1}\right)$, and $R_{o}$ is the reference resistance $(\Omega)$.

\section{RESULTS AND ANALYSIS}

The authors of this paper did not have a selective body of precisely controlled temperature and know emissivity, a black body was used as the reference object of temperature measurement. However, the black body was tested as an object of unknown emissivity to simulate real measurement mode and task of the system. Additionally, a known detector data was used to facilitate the simulation of the real measurement process. The results of the system are discussed below. The given detector data are as follows: Heat transfer coefficient, $U=2 \times 104 \mathrm{~W} \mathrm{~m}^{-2}{ }^{\circ} \mathrm{C}^{-1}$, detector surface area, $A=1.6 \times 10^{-4} \mathrm{~m}^{2}$, heat capacity, $M C=1.6 \times 10^{-2} \mathrm{~J}^{\circ} \mathrm{C}^{-1}$, temperature coefficient of resistance, $\alpha=0.004{ }^{\circ} \mathrm{C}^{-1}$, reference resistance, $R_{o}=100 \Omega$ at $0{ }^{\circ} \mathrm{C}$.

The temperature range of the source is given as $900{ }^{\circ} \mathrm{C}-1200{ }^{\circ} \mathrm{C}$, the temperature of the surrounding environment is taken as 25 ${ }^{\circ} \mathrm{C}$. The radiant energy from the process falls on the detector at a rate given by Equation (4). For the given source temperature range, the maximum and minimum incident power on the detector can be computed using the rate at which the radiant energy falls on the detector as:

$$
\begin{aligned}
P_{\text {Dmax }}= & 50 \times 10^{-12} \times(1200+273)^{4} \\
& =50 \times 10^{-12} \times 1473^{4} \\
& =235.39 \mathrm{~W} \\
P_{\text {Dmin }}= & 50 \times 10^{-12} \times(900+273)^{4} \\
& =50 \times 10^{-12} \times 1173^{4} \\
& =94.66 \mathrm{~W}
\end{aligned}
$$

Similary, Equation (7) was used to obtain the corresponding $\mathrm{T}_{\mathrm{Dmax}}$ and $\mathrm{T}_{\mathrm{Dmin}}$ as thus:

$$
\begin{aligned}
\mathrm{T}_{\text {Dmax }}= & \frac{235.39}{2 \times 10^{4} \times 1.6 \times 10^{-4}}+25 \\
& =73.56+25 \\
& =98.56^{\circ} \mathrm{C} \\
\mathrm{T}_{\text {Dmin }} & =\frac{94.66}{2 \times 10^{4} \times 1.6 \times 10^{-4}}+25 \\
& =29.54+25 \\
& =54.58^{\circ} \mathrm{C}
\end{aligned}
$$

Now, since $R \gg>R_{o}$ and for design purposes, $R$ can be chosen to be approximately $=10 \mathrm{~K} \Omega$. For the detector to be within $2 \%$ of equilibrium when the chopper (shutter) switches, the relationship below must be satisfied. i.e:

$$
\tau_{\mathrm{D}}<\frac{1}{6 f_{c}}
$$

where denotes the time constant of the detector (usually in $\mathrm{ms}$ ) and $f_{c}$ represents the chopping frequency of the rotating shutter. Using Equation (8), the detector time constant is computed as: 
International Journal of Advances in Scientific Research and Engineering (ijasre), Vol 6 (11), November -2020

$$
\begin{aligned}
\tau_{D} & =\frac{1.6 \times 10^{-2}}{2 \times 10^{4} \times 1.6 \times 10^{-4}} \\
\tau_{D} & =5 \times 10^{-3} \mathrm{~s} \\
\tau_{D} & =5 \mathrm{~ms}
\end{aligned}
$$

Therefore, recall Equation (17) and substituting the value of the time constant yields:

$$
\begin{aligned}
& 5 \mathrm{~ms}<\frac{1}{6 f_{c}} \\
& 30 \mathrm{~ms}<\frac{1}{f_{c}} \\
& f_{c}<\frac{1}{30 \mathrm{~ms}} \\
& f_{c}<33.33 \mathrm{~Hz}
\end{aligned}
$$

Therefore, choosing $f_{c}$ so the above relationship is satisfied, so $f_{c}=30 \mathrm{~Hz}$ which effectively means that the shutter is rotating at $1800 \mathrm{rpm}$. Now, the pulse duration time for the shutter cycles is thus:

$$
\begin{aligned}
& \mathrm{T}_{\mathrm{p}}=\frac{1}{30} \\
& \mathrm{~T}_{\mathrm{p}}=33.33 \mathrm{~ms}
\end{aligned}
$$

This means that, since the detector has the behavior of a first order system, the detector will have enough time to settle before the next shutter transition. This is illustrated in the Figure 4 below.

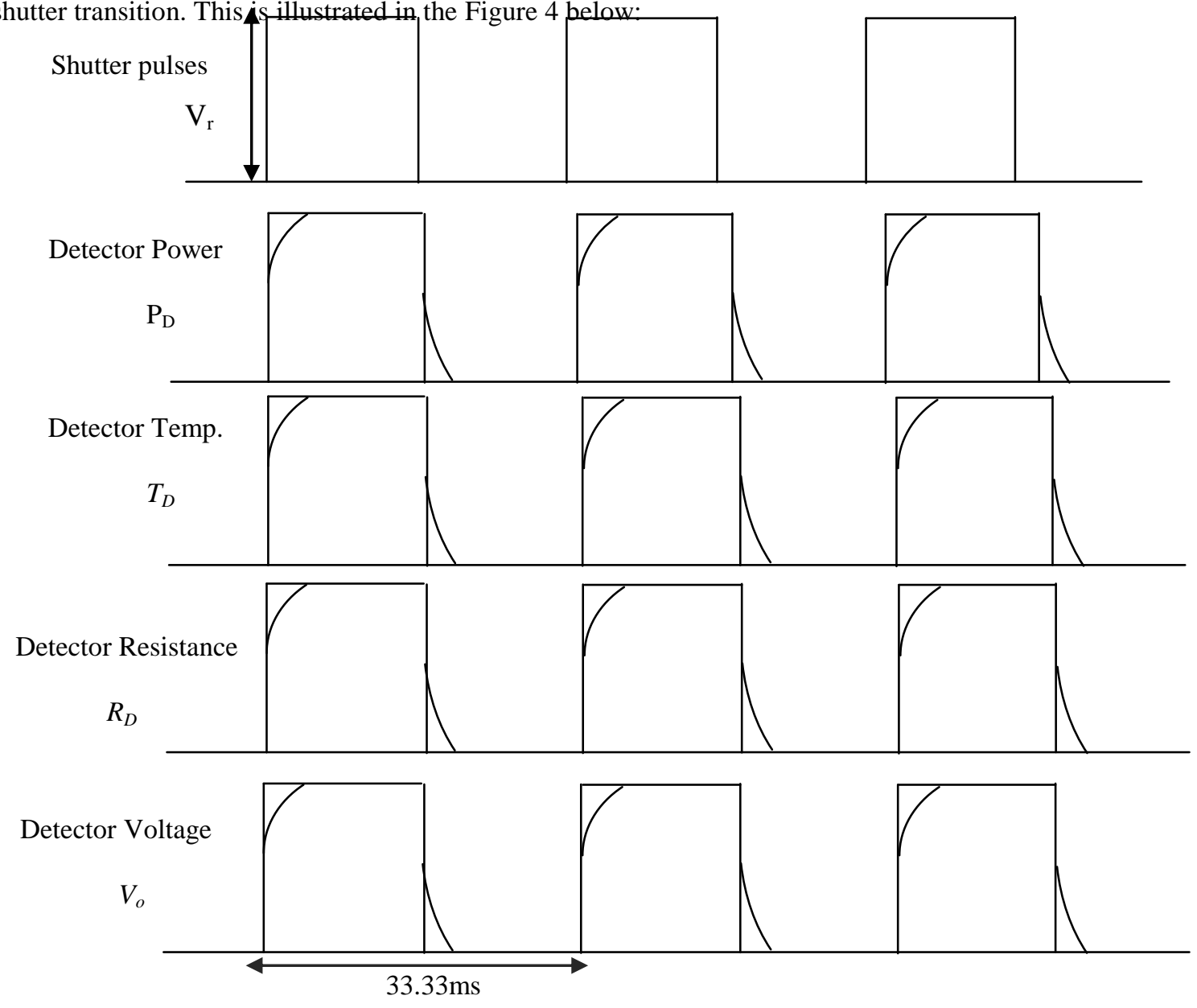

Figure 4: Shape of the shutter pulses and the detector behaviour

Figure 4 shows how the detector tries to catch up with the shutter pulses and settles before the next shutter transition. 
The maximum output voltage of the detector $V_{\text {omax }}$ occurs when $P_{D}$ and $T_{D}$ are maximum and this corresponds to the amplitude of the chopper (shutter) pulses. Recall Equation (15) thus:

$$
V_{\text {omax }} \approx V_{S} \alpha \frac{R_{o}}{R}\left(T_{D \max }-T_{S}\right)
$$

Since the output of the detector (bridge output) is very small, as a result is going to be very susceptible to environmental noise, a PSD is used which can be regarded as a multiplier or demodulator plus a low pass filter and form the basis of a lock in amplifier. Using the PSD, the effects of noise can be substantially reduced, enabling small signals to be recovered from an extremely noisy environment. The PSD output using square wave reference signal is:

$$
V_{P S D}=\frac{2 V V_{r}}{\pi} \cos \theta
$$

where $V_{r}$ denates the amplitude of the shutter pulse $=V_{\text {omax }}$ (at maximum temp.) Now, $V_{\text {omax }}$ from Equation(18) and substituting $\mathrm{T}_{\text {Dmax }}$ computed above, and $V_{S}=0.707 \mathrm{~V}$ (rms value):

$$
\begin{array}{r}
V_{\text {omax }}=0.707 V x(0.004) \frac{100}{10000}(98.56-25) \\
V_{\text {omax }}=2.08 \times 10^{-3} \times V(V)
\end{array}
$$

Substituting $V_{\text {omax }}$ into Equation (19) and assuming $\theta={ }^{\circ} 0$ for maximum output, which should be less than or equal to $1 \mathrm{~V}$ (ADC maximum range):

$$
\begin{aligned}
V_{P S D} & =\frac{2 \times v \times 0.707 v \times 2.08 \times 10^{-3}}{\pi} \leq 1 \\
V^{2} & =\frac{\pi}{2 \times 2.08 \times 10^{-3}} \\
V^{2} & =755.19 \\
\therefore & V=27.48 \mathrm{~V}
\end{aligned}
$$

For design purposes, assuming $V=21.5 \mathrm{v}$

$$
\begin{aligned}
\therefore \quad V_{S} & =0.707 \times 21.5 \\
& =15.2005 \mathrm{~V} \\
& \sim=15 \mathrm{~V}
\end{aligned}
$$

Therefore, by Equation (20) the maximum detector voltage (bridge output) $V_{\text {omax }}$ which corresponds to the amplitude of the shutter pulse $V_{r}$ when the detector temperature is maximum.

$$
\begin{aligned}
V_{\text {omax }}= & 2.08 \times 10^{-3} \times \mathrm{V}(\mathrm{V})=V_{r} \\
& =2.08 \times 10^{-3} \times 21.5 \\
& =44.13 \mathrm{mV}
\end{aligned}
$$

Similarly, by Equation (19) the maximum voltage output of the phase sensitive detector (PSD) is thus:

$$
V_{P S D \max }=\frac{2 V V_{r}}{\pi} \cos \theta
$$


International Journal of Advances in Scientific Research and Engineering (ijasre), Vol 6 (11), November -2020

$$
\begin{aligned}
& V_{P S D \max }=\frac{2 \times 21.5 \times 44.13 \times 10^{-3}}{\pi} \cos (0) \\
& V_{P S D \max }=\frac{1.8976}{\pi} \\
& V_{P S D \max }=0.60 \mathrm{~V}
\end{aligned}
$$

This $V_{P S D}$ is the voltage going into the ADC digitizer. Using Equation (4), Equation (7), Equation (19), and Equation (20), and substituting where applicable $V_{s}=15 \mathrm{~V}, R=10 \mathrm{~K} \Omega$, the values of $T_{D}, V_{o}$ (output of the bridge), $P_{D}$ and $V_{P S D}$ are computed and tabulated as shown in Table 1.

Table 1: Design result for broadband pyrometer system

\begin{tabular}{|c|c|c|c|c|c|}
\hline$T\left({ }^{\circ} C\right)$ & $T(K)$ & $P_{D}(W)$ & $T_{D}\left({ }^{o} C\right)$ & $V_{o}(m V)$ & $V_{P S D}$ \\
\hline 900 & 1173 & 94.659031 & 54.5809471 & 17.7486 & 0.2429 \\
\hline 915 & 1188 & 99.594594 & 56.1233107 & 18.674 & 0.25556 \\
\hline 930 & 1203 & 104.72069 & 57.725217 & 19.6351 & 0.26872 \\
\hline 945 & 1218 & 110.04217 & 59.388179 & 20.6329 & 0.28237 \\
\hline 960 & 1233 & 115.56393 & 61.1137288 & 21.6682 & 0.29654 \\
\hline 975 & 1248 & 121.29094 & 62.9034173 & 22.7421 & 0.31124 \\
\hline 990 & 1263 & 127.22821 & 64.7588147 & 23.8553 & 0.32647 \\
\hline 1005 & 1278 & 133.38083 & 66.6815098 & 25.0089 & 0.34226 \\
\hline 1020 & 1293 & 139.75395 & 68.6731106 & 26.2039 & 0.35861 \\
\hline 1035 & 1308 & 146.35278 & 70.7352442 & 27.4411 & 0.37555 \\
\hline 1050 & 1323 & 153.18258 & 72.8695564 & 28.7217 & 0.39307 \\
\hline 1065 & 1338 & 160.24868 & 75.0777122 & 30.0466 & 0.4112 \\
\hline 1080 & 1353 & 167.55647 & 77.3613955 & 31.4168 & 0.42996 \\
\hline 1095 & 1368 & 175.11139 & 79.7223092 & 32.8334 & 0.44934 \\
\hline 1110 & 1383 & 182.91896 & 82.1621752 & 34.2973 & 0.46938 \\
\hline 1125 & 1398 & 190.98475 & 84.6827343 & 35.8096 & 0.49007 \\
\hline 1140 & 1413 & 199.31439 & 87.2857464 & 37.3714 & 0.51145 \\
\hline 1155 & 1428 & 207.91357 & 89.9729904 & 38.9838 & 0.53351 \\
\hline 1170 & 1443 & 216.78804 & 92.746264 & 40.6478 & 0.55629 \\
\hline 1185 & 1458 & 225.94363 & 95.6073841 & 42.3644 & 0.57978 \\
\hline 1200 & 1473 & 235.3862 & 98.5581865 & 44.1349 & 0.60401 \\
\hline
\end{tabular}

\section{i. Calibration}

From Table 1, the calibration graph of the output voltage $\mathrm{V}_{\mathrm{PSD}}$ versus the source temperature is depicted in Figure 5. This is done in order to interpret the output voltage of the pyrometer in terms of the temperature. The calibration of the pyrometer from experiment is necessary because the output of the pyrometer is affected considerably by losses due to the surface condition at the face of the detector, the variation of emissivity of the object with its surface temperature, and other factors. 


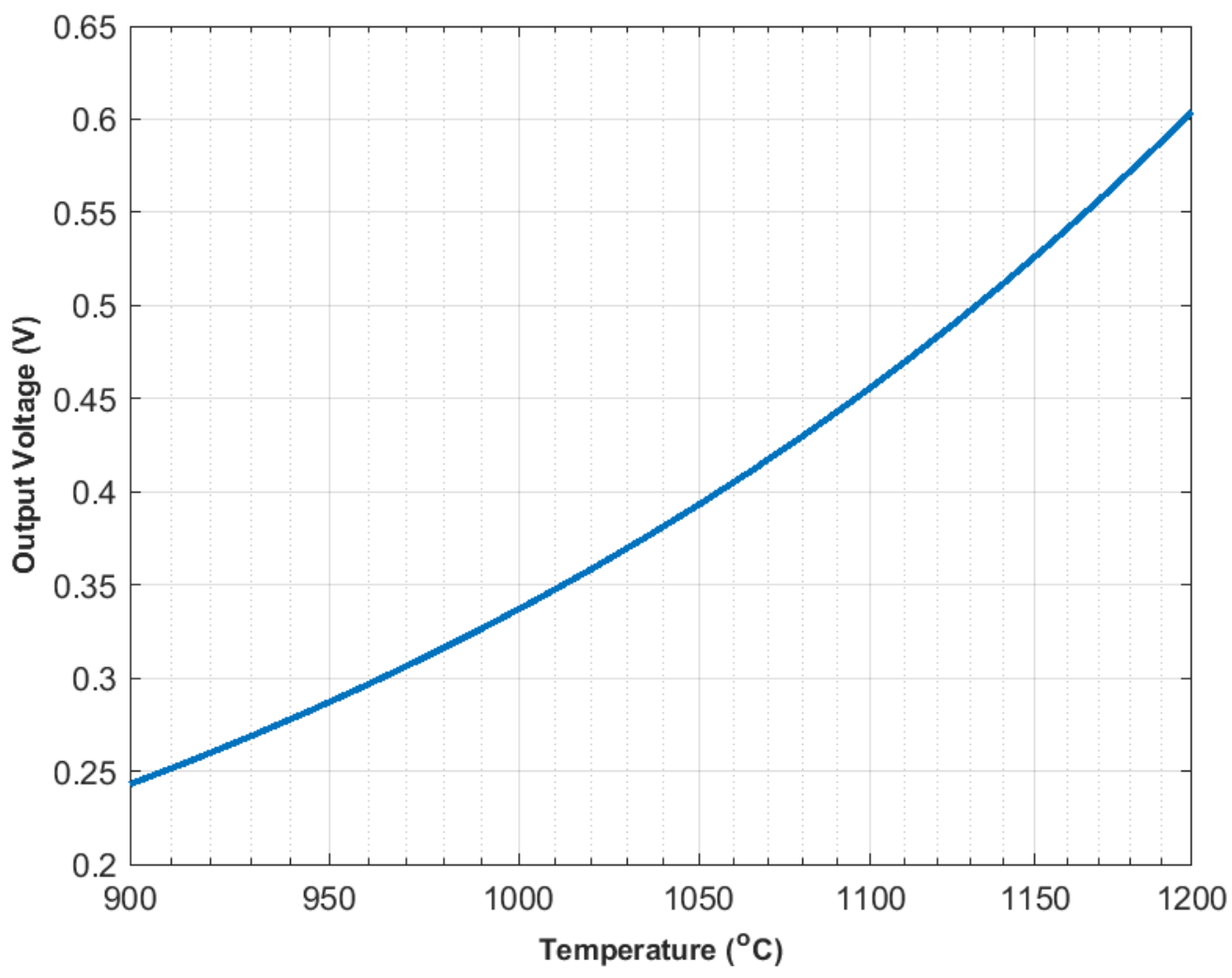

Figure 5: Calibration curve chopped broadband radiation pyrometer

However, the advantage of calibration with the black body is that high temperature accuracy measurements can be obtained with the pyrometer system. The main problem, however, is that the temperature of the black body is limited to $1200{ }^{\circ} \mathrm{C}$. The measurement of temperature higher than $1200{ }^{\circ} \mathrm{C}$ is possible if theoretical calibration curve is calculated. This can be obtained using the Planck's law or Wien's law with the knowledge of the detector responses and spectral transmissions of the optical components.

Additionally, it can be seen from Figure 5 that the pyrometer temperature range configuration has approximately $0.60 \mathrm{~V}$ corresponding to the maximum temperature and a minimum of approximately $0.24 \mathrm{~V}$ corresponding to the minimum temperature in the measurement range. Furthermore, the system resolution spans in the range $-1 \mathrm{~V}$ to $+1 \mathrm{~V}$, which implies that my pyrometer configuration has limited the system to the range $0 \mathrm{~V}$ to $1 \mathrm{~V}$ as the configuration, has no negative value of voltage (i.e resolution compromised).

Interestingly, the pyrometer system accuracy based on linearity can be deduced from Equation (6) at steady state. The relationship between the incident power of the detector and the detector temperature is shown in Figure 6. 


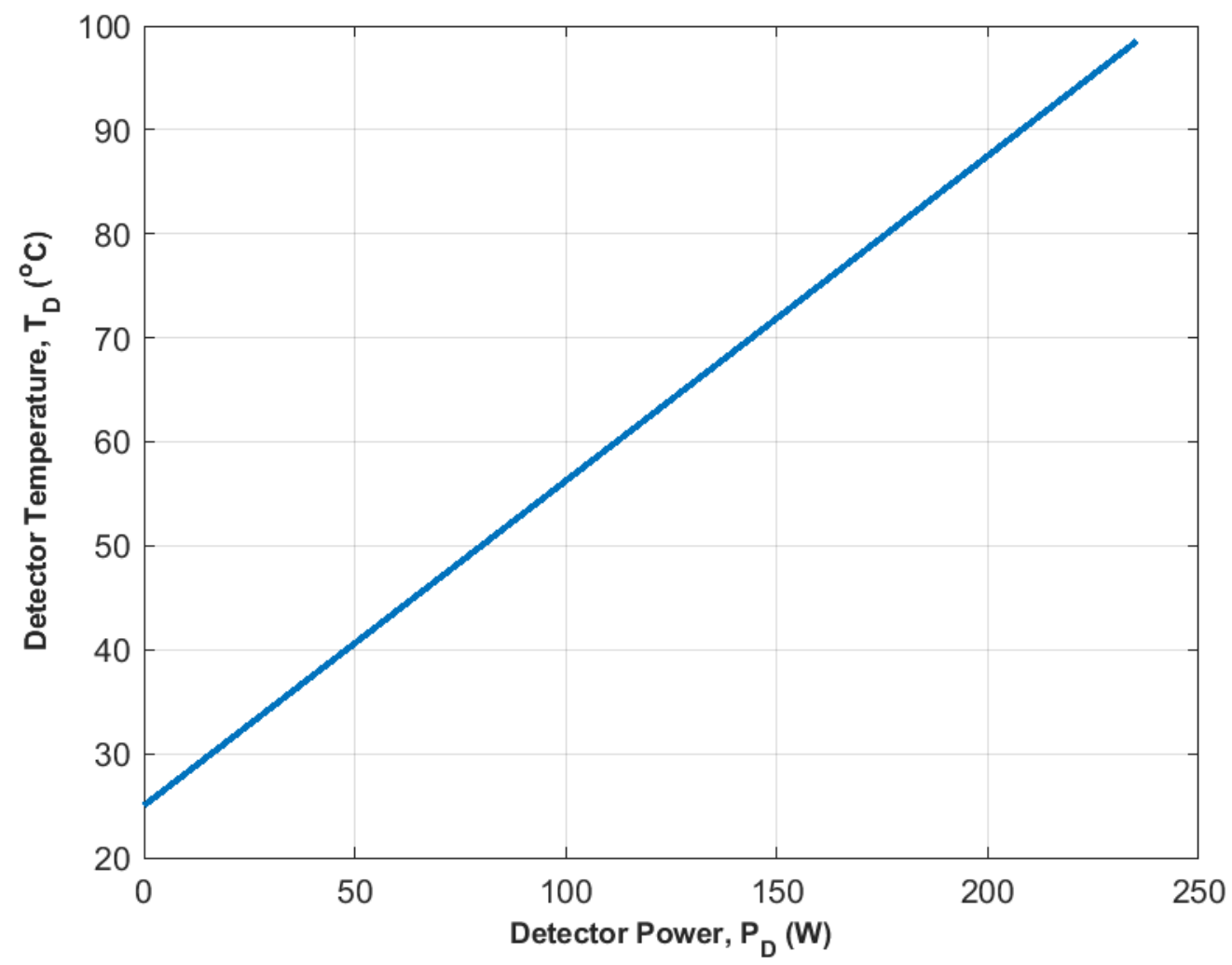

Figure 6: Linear characteristics chopped broadband pyrometer at steady state

As shown in Figure 6, the intercept on the $T_{D}$ axis corresponds to the surrounding temperature $T_{S}$. Hence, a linear function $T_{D}=0.3125 P_{D}+25$ is obtained from the graph, where $0.3125\left(w^{-1}{ }^{\circ} \mathrm{C}\right)$ is the sensitivity which corresponds to the $U A$ relationship in Equation (7).

\section{CONCLUSION}

In summary, the signal conditioning and acquisition elements for a chopped broadband radiation pyrometer for temperature measurements in the range $900{ }^{\circ} \mathrm{C}$ to $1200{ }^{\circ} \mathrm{C}$ have been designed. A bolometer was used as the detector which is a passive resistive device, hence a bridge circuit was used to produce an output voltage proportional to the temperature (i.e17.75 $\mathrm{mV}$ to $44.13 \mathrm{mV}$ ) corresponding to the temperature range of the pyrometer. Since this output is very small, it implies that, is very susceptible to noise and a PSD was used in the form of lock- in amplifier plus low pass filter which amplifies, recover the signal and produce an output within the range of the digitizer (i.e $0.24 \mathrm{~V}-0.60 \mathrm{~V}$ ) corresponding to the temperature range. It was also shown that a suitable chopping frequency of $30 \mathrm{~Hz}$ was chosen for the shutter which has been verified to be within the physical properties of the system by satisfying the relationship: $\tau_{\mathrm{D}}<\frac{1}{6 f_{C}}$ in order for the detector to have enough time to settle before the next shutter transition as shown in Figure 4. Calibration graph was also provided giving the relationship of the output voltage and the temperature which is approximately linear.

\section{REFERENCES}

1. Bentley, J. P. 2005. Principles of Measurement Systems, Fourth Edition, Prentice Hall, USA. ISBN: 978 - 0 - 13043028 -1 .

2. Jack, E. K., Etu, I., and Ukanide, N. V. 2016. The Design of Signal Conditioning and Acquisition Elements of Chopped Broadband Radiation Pyrometer. In Proceeding of the World Congress on Engineering and Computer Science, Vol. 1.

3. Alan, M. S. 2001. Measurement and Instrumentation Principles, Third Edition, Butterworth-Heinemann, Uk.

4. Bieleck, Z. and Chrzanowski, K. 1999. Infrared Pyrometer for Temperature Measurement of Objects for both Wavelength and Time Dependent Emissivity. Optica Applicata, Vol. XXIX (3), Pp. 285 - 292.

5. Muller, B., and Renz, U. 2001. Development of Fast Fiber - Optic Two Color Pyrometer for the Temperature Measurement of Surfaces with Varying Emissivities. Review of Scientific Instruments, Vol. 72 (8), Pp. 3366 - 3374. 
6. Ng, D., and Fralick, G. 2001. Use a Multiwavelength Pyrometer in Several Elevated Temperature Aerospace applications. Review of Scientific Instruments, Vol. 72 (1), Pp. 1522 - 1530.

7. Ueda, T., Hosokawa, A., and Yamamoto, A. 1985. Studies on Temperature of Abrasive Grains in Grinding - Application of Infrared Radiation Pyrometer. Journal of Engineering for Industry, Transactions of the ASME, Vol. 107, Pp. 127 133.

8. Vàzquez, C., Pérez-Prieto, S., López-Cardona, D. J., Tapetado, A., Blanco, E., Moreno-López, J., Montero, S. D. and Lallana, C. P. 2018. Fiber-Optic Pyrometer with Optically Powered Switch for Temperature Measurement. Sensors, Vol. 18 (483), Pp. 1 - 10.

9. Wallace, P. 2010. Instrumentation for Mechanical Condition Monitoring: Module Notes 